\title{
Transmission Cooperative Strategies for MIMO-OFDM Heterogeneous Networks
}

\author{
Syed Saqlain ALI, Daniel CASTANHEIRA, Adão SILVA, Atilio GAMEIRO \\ DETI, Instituto de Telecomunicações, University of Aveiro, Portugal \\ syedsaqlain@av.it.pt, dcastanheira@av.it.pt, asilva@av.it.pt,amg@ua.pt
}

\begin{abstract}
Mobile traffic in cellular networks is increasing exponentially, mainly due to the use of data intensive services like video. One way to cope with these demands is to reduce the cell-size by deploying small-cells along the coverage area of the current macro-cell system. The deployment of small-cells significantly improves indoor coverage. Nevertheless, as additional spectrum licenses are difficult and expensive to acquire it is expected that the macro and small-cells will coexist under the same spectrum. The coexistence of the two systems results in crosstier/inter-system interference. In this context, we design several interference alignment based techniques for the downlink of heterogeneous networks, in order to cancel the interference generated from macro-cell at small-cell user terminals. More specifically, in this contribution we design interference alignment methods under different levels of inter-system coordination and the constraint that the performance of macro-cell system is kept close to the case where small-cell system is switched-off. Numerical results demonstrate that the proposed methods achieve close to the optimal performance with low overhead.
\end{abstract}

\section{Keywords}

Interference alignment, space-frequency block codes, heterogeneous networks, small-cells, MIMO-OFDM, downlink.

\section{Introduction}

The proliferation of wireless devices, such as smart phones, mobile broadband modems, tablets, and mobile data applications, will in the near future be overwhelming wireless networks. The expected increasing demand for high data rates has urged mobile operators to explore new ways in order to improve the coverage and provide boost in the network capacity [1]. Cell reduction concept is one of the key enabling technologies to increase the network capacity as demanded for the evolution of mobile technologies towards 5G. Network densification through use of small cells (SCs) has been considered in 3GPP which made small cells an integral part of LTE Advanced by developing the concept of heterogeneous networks (HetNets)
[2]-[5]. Small cells are formed by low-power and reducedsize wireless access points that operate in licensed spectrum and are operator-managed. They operate inside the coverage area of a macro-cell, creating a heterogeneous network, and offer great advantages for operators and for users, who get better coverage and higher data rate, and can access new services [6]. Another key aspect in next generation cellular networks is the spectral efficiency that is becoming more and more important in order to utilize the limited spectrum efficiently. Therefore, to ensure fair and economical use of available spectrum, relevant standardization bodies have established spectrum allocation policies. However, several measurement studies reveal that the fixed spectrum policies led to inefficient spectrum utilization [7]. Moreover, it indicates that there is space to improve the spectrum utilization process.

As frequency is a scarce resource and acquiring spectrum licenses for small-cells is difficult and expensive, it is preferred that the small-cells share the frequency band with the macro-cell system. However, this results in a number of challenges namely related with interference management [8]. Not only should the interference between the macrocell and small-cells be considered, but also the interference among the small cells introduces a complex problem [9], [10]. The macro-cell system is the owner of the spectrum license and in the literature of cognitive radio (CR) [11][13] the macro-cell terminals are denominated as primary users. Similarly, the small-cell terminals use the spectrum of the former in an opportunistic way and are denominated secondary. In this context, the wireless networks require more careful and dynamic planning and if these two systems are not carefully designed then it will cause harmful interference, hence degrading the performance of both macro-cell and small-cell systems [14]. In order to deal with interference issues in heterogeneous networks different interference management techniques have been proposed [15]-[17]. Also multiple antenna techniques in combination with $\mathrm{CR}$ have been considered in some publications [18], [19].

In [20] the authors have considered an underlay CR uplink scenario, where both primary and secondary systems have two antennas. Secondary users use the two antennas to insert a null in the direction of primary BS and protect the primary users from secondary interference. On the other hand, two antennas are used at primary users in 
order to perform beam steering in the direction of the primary BS. One recent and effective solution to deal with interference in two tiered networks is the interference alignment (IA) [21] technique. The concept of IA has emerged as a key approach to align an arbitrary large number of interferers and achieve the maximum degree of freedom (DoF) in wireless networks [22], [23]. In [23] the authors proposed a new interference alignment scheme for heterogeneous networks with multiple antennas. The scheme proposed successively creates transmit beamforming vectors for the small-cell BSs (SBSs) and for the macro BS (MBS) assuming that they have different number of transmit antennas.

Furthermore, the concept of IA has been jointly used with $\mathrm{CR}$ in order to mitigate interference in two-tiered networks [24], [25]. In [24] the authors proposed a novel overlay cognitive interference alignment scheme to increase the spectral efficiency of the two-tiered networks. In [25] a novel cross-tier IA scheme in conjunction with CR to mitigate the interference in heterogeneous networks was derived. In [26] a joint IA and cognitive communication technique in order to mitigate interference of small-cell user terminals (UTs) towards the macro base station was proposed. The authors proposed three IA methods with different levels of inter-system information exchange: coordinated, static and uncoordinated approaches. The coordinated method achieves the best performance but has very high inter-system information exchange requirements while on the other hand the uncoordinated method requires no information exchange but the performance is degraded. Therefore, to overcome the shortcomings of coordinated and uncoordinated methods, the authors in [27] proposed a coordinated one-bit method for the uplink of heterogeneous networks. In [28] the methods mentioned in [27] were extended for the downlink of a heterogeneous network.

The amount of information that needs to be exchanged between the cooperating identities is a key aspect in coordinated based networks as it defines the overhead needed by the network to get the benefits from cooperation. If full information exchange is available between the macro-cell and small-cells, the system achieves the best performance at the expense of a high overhead while where no information is exchanged between the two systems, there is no overhead but the system experiences the worst performance as discussed in [26]-[28]. The development of schemes that can provide close to optimal performance with limited information exchange and without any intersystem coordination is therefore of paramount importance. Therefore, the main contributions of this work are the following

- Extension of the IA schemes proposed in [28] for a specific antenna configuration scenario, to a more generic antenna configuration in the downlink of heterogeneous networks.

- Proposal of a new efficient IA scheme under limited information exchange to tackle the interference of macro-cell system at small-cell UTs (SUTs). Namely, only a quantized version of the alignment direction is fed back from MBS to the small-cell system.

- Proposal of new joint IA and space-frequency block codes (SFBC) based schemes that allows efficient spectral coexistence of the two systems without any inter-system information-exchange requirements.

The rest of the paper is organized as follows: Section 2 introduces the system and signal models. In Sec. 3, we start by summarizing the related work and then the proposed schemes are derived in detail. In Sec. 4, we present the numerical results and performance comparison of the proposed methods with existing ones. Finally, conclusions are provided in Sec. 5.

Notations: Bold upper case letters denote matrices, bold lower case letters denote vectors. The operations $(.)^{H}$ and (.) ${ }^{*}$ stands for the Hermitian transpose and conjugate of a matrix, respectively. null(A) denotes a matrix whose columns span the null-space of matrix (A) and $\mathbf{A}=$ $\operatorname{diag}\left(\mathbf{A}_{1}, \mathbf{A}_{2}\right)$ a diagonal matrix with entries $\mathbf{A}_{1}$ and $\mathbf{A}_{2} . \mathbf{A}_{(n)}$ denotes the column $n$ of matrix $\mathbf{A}$ and $\mathbf{A}_{(m, n)}$ the entry at row $m$ and column $n$ of matrix A. $\sigma^{2}$ denotes the noise variance per subcarrier and $\mathbf{I}$ denotes the identity matrix.

\section{System Model}

We consider the downlink of a two-tiered network with $K$ small-cells overlaid within the coverage region of macro-cell and sharing the same spectrum. Furthermore, the small-cell base stations (SBSs) are able to cooperate through a backhaul network (e.g Radio over Fiber) to a central unit $(\mathrm{CU})$ that allows joint processing of transmitted signals as depicted in Fig. 1. We consider the downlink scenario, i.e. both MBS and SBSs transmit data to the corresponding UTs. We consider that the MBS serves only one user terminal, macro UT (MUT), per sub-

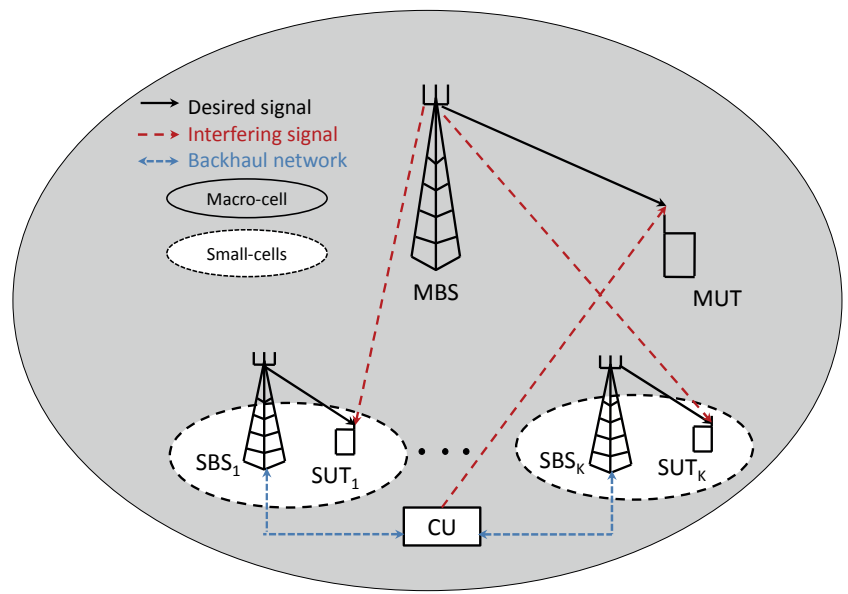

Fig. 1. System model: Small-cells within the coverage area of macro-cell. 


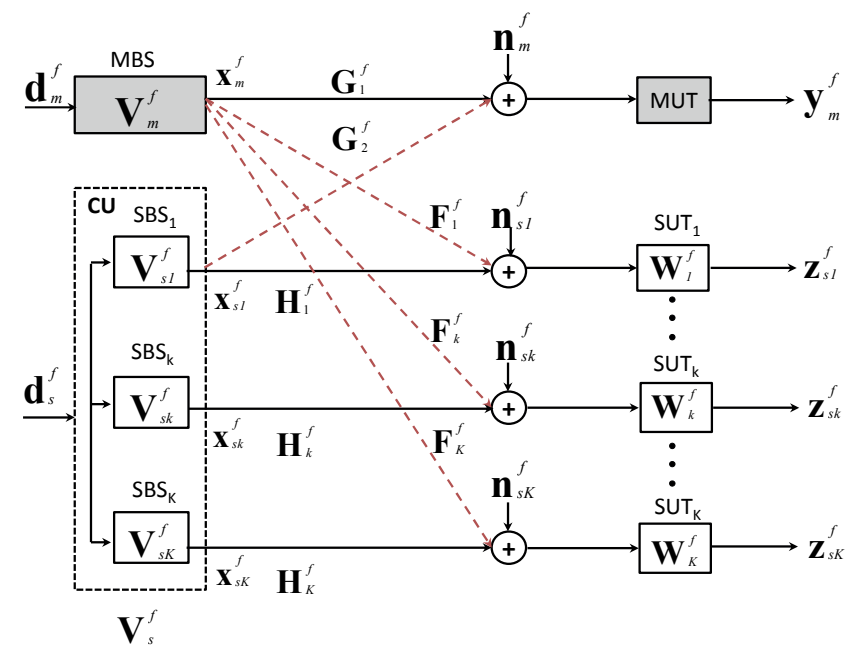

Fig. 2. Block diagram of considered system.

carrier $^{1}$, and the SBS $k$ serves only the small-cell user terminal $k\left(S U T_{k}\right) i=\{1, \ldots, K\}$. Furthermore, we assume OFDM based terminals with $N_{c}$ subcarriers, where the transmit power per subcarrier of MBS and SBSs is constraint to $P_{m}$ and $P_{s}$, respectively.

\subsection{Signal Model without SFBC}

In this section, we describe the signal model of the macro-cell and small-cell systems for the case where no space-frequency coding is employed at the MBS. The block diagram of the considered systems is shown in Fig. 2.

\subsubsection{Macro-Cell System}

At the macro-cell system we assume that the MBS and MUT have $M_{m}$ and $N_{m}$ antennas, respectively. The transmitted signal $\left(\mathbf{x}_{m}^{f}\right)$ at the MBS on subcarrier $f$ is given by

$$
\mathbf{x}_{m}^{f}=\gamma_{m}\left(\mathbf{V}_{m}^{f} \mathbf{d}_{m}^{f}\right)
$$

where $\gamma_{m}^{2}=P_{m} / \operatorname{tr}\left(\mathbf{V}_{m}^{f H} \mathbf{V}_{m}^{f}\right), \mathbf{V}_{m}^{f} \in \mathbb{C}^{M_{m} \times N_{m}}$ and $\mathbf{d}_{m}^{f} \in \mathbb{C}^{N_{m}}$ denote a normalizing constant, the precoder and the transmitted symbols at the MBS, respectively.

The received signal in the frequency domain at the $\operatorname{MUT}\left(\mathbf{y}_{m}^{f} \in \mathbb{C}^{N_{m}}\right)$ can be mathematically expressed as

$$
\mathbf{y}_{m}^{f}=\mathbf{G}_{1}^{f} \mathbf{x}_{m}^{f}+\mathbf{G}_{2}^{f} \mathbf{x}_{s}^{f}+\mathbf{n}_{m}^{f}
$$

where

$\mathbf{x}_{s}^{f} \in \mathbb{C}^{M_{s} K}, \mathbf{G}_{1}^{f} \in \mathbb{C}^{N_{m} \times M_{m}}, \mathbf{G}_{2}^{f} \in \mathbb{C}^{N_{m} \times M_{s} K}$ and $\mathbf{n}_{m}^{f} \in \mathbb{C}^{N_{m}}$, denote the overall transmitted signal at small-cells, the channel between MBS and MUT, the overall channel

1 Considering an OFDM/A based system, the total number of macro-cell users can be significantly larger than one, since different set of resources can be allocated to different users. between CU and MUT (i.e., the channels between the SBSs and the MUT) and the zero mean white Gaussian noise with variance $\sigma^{2}$, respectively. We consider that at the macro-cell BS only $\mathbf{G}_{1}^{f}$ is known and it has no information about the small-cell system. Furthermore, we assume that the MUT is a mobile terminal and then $\mathbf{G}_{1}^{f}$ and the precoder $\mathbf{V}_{m}^{f}$ (function of macro-cell channel $\mathbf{G}_{1}^{f}$ ) changes on every Transmission Time Interval (TTI).

\subsubsection{Small-Cell System}

In the small-cell system, each SBSs has $M_{s}$ transmit and the $S U T_{k} k=\{1, \ldots, K\}$ has $N_{s}$ receive antennas. The transmitted signal $\left(\mathbf{x}_{s}^{f}\right)$ at the $\mathrm{CU}$ on subcarrier $f$ is expressed as

$$
\mathbf{x}_{s}^{f}=\gamma_{s}\left(\mathbf{V}_{s}^{f} \mathbf{d}_{s}^{f}\right)
$$

where $\mathbf{V}_{s}^{f} \in \mathbb{C}^{M_{s} K \times\left(\mathbb{N}_{s}-N_{m}\right) K}, \quad \mathbf{d}_{s}^{f}=\left[\mathbf{d}_{s k}^{f}\right]_{1 \leq k \leq K} \in \mathbb{C}^{\left(N_{s}-N_{m}\right) K}$, $\mathbf{d}_{s k}^{f} \in \mathbb{C}^{N_{s}-N_{m}}$ and $\gamma_{s}^{2}=P_{s} / \operatorname{tr}\left(\mathbf{V}_{s}^{f H} \mathbf{V}_{s}^{f}\right)$ denote the overall precoder computed at the $\mathrm{CU}$, the concatenation of the $K$ SBSs transmit symbols, the SBS $k$ transmit symbols and a normalizing constant.

The received signal after the filter matrix $\left(\mathbf{W}_{k}^{f}\right)$ at the $S U T_{k}$ is

$$
\mathbf{z}_{s k}^{f}=\mathbf{W}_{k}^{f}\left(\mathbf{F}_{k}^{f} \mathbf{x}_{m}^{f}+\mathbf{H}_{k}^{f} \mathbf{x}_{s}^{f}+\mathbf{n}_{s k}^{f}\right)
$$

where $\mathbf{F}_{k}^{f} \in \mathbb{C}^{N_{s} \times M_{m}}, \mathbf{H}_{k}^{f} \in \mathbb{C}^{N_{s} \times M_{s} K}$ and $\mathbf{n}_{s k}^{f} \in \mathbb{C}^{N_{s}}$ denote the channel between the MBS and $S U T_{k}$, the overall channel between the SBSs and $S U T_{k}$ and the zero mean white Gaussian noise with variance $\sigma^{2}$ at $S U T_{k}$, respectively. In the following, we assume that the SUTs are low mobility terminals ${ }^{2}$ and then the channel $\mathbf{F}_{k}^{f}$ can be considered as quasi-static which reduces the overhead required for their estimation.

\subsection{Signal Model with SFBC}

In this section, we describe the signal model for the case where space-frequency coding is performed at the MBS. Two SFBC are considered: the standard Alamouti [29] with the data symbols coded in space and frequency as shown in Fig. 3, and the double-SFBC based scheme [30]. The latter one is an extension of the standard two transmit

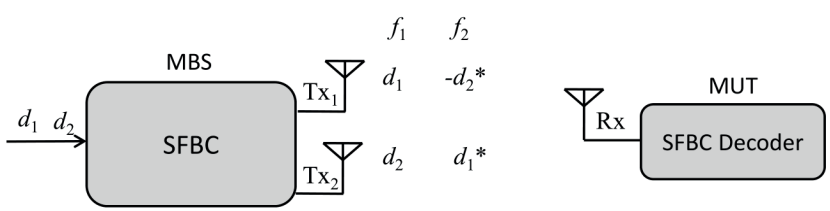

Fig. 3. SFBC scheme at MBS.

2 Since the terminals associated to the small-cells are mainly indoor/pedestrian users. 
antenna SFBC to 4 transmit antennas, i.e., two pairs of data symbols are transmitted in parallel over 2 subcarriers, as shown in Fig. 4. Furthermore, from the context of spacetime/space-frequency coding literature, the channel between two adjacent carriers is assumed to be approximately constant ${ }^{3}$, i.e. $\mathbf{G}_{1}^{f_{1}} \approx \mathbf{G}_{1}^{f_{2}}$.

\subsubsection{Macro-Cell System with SFBC Scheme}

In this case we employ the standard Alamouti SFBC based scheme at the macro-cell system, assuming that the transmitter has $M_{m}=2$ antennas and the receiver a single antenna, $N_{m}=1$. In this well-known method the encoder takes a block of two data symbols, i.e. $d_{1}, d_{2}$. At a given subcarrier, two symbols are simultaneously transmitted from the two antennas, as shown in Fig. 3. For the first subcarrier $f_{1}$ the symbol transmitted from the first antenna is denoted by $d_{1}$ and from the second one by $d_{2}$ and over subcarrier $f_{2},\left(-d_{2}\right)^{*}$ and $\left(d_{1}\right)^{*}$ are transmitted from the first and second antennas, respectively. The transmitted signal at the MBS on subcarriers $f_{1}\left(\mathbf{x}_{m}^{f_{1}}\right)$ and $f_{2}\left(\mathbf{x}_{m}^{f_{2}}\right)$ is given by

$$
\mathbf{x}_{m}^{f_{1}}=\left[\begin{array}{l}
d_{1} \\
d_{2}
\end{array}\right], \quad \mathbf{x}_{m}^{\left(f_{2}\right)^{*}}=\left[\begin{array}{c}
-d_{2} \\
d_{1}
\end{array}\right] .
$$

From (5) the received signal at MUT on subcarrier $f_{1}\left(\mathbf{y}_{m}^{f_{1}}\right)$ is given by

$$
\mathbf{y}_{m}^{f_{1}}=\mathbf{G}_{1(1)}^{f_{1}} d_{1}+\mathbf{G}_{1(2)}^{f_{1}} d_{2}+\mathbf{G}_{2}^{f_{1}} \mathbf{x}_{s}^{f_{1}}+\mathbf{n}_{m}^{f_{1}}
$$

and at subcarrier $f_{2}$ (after applying the conjugate operator) the received signal $\mathbf{y}_{m}^{\left(f_{2}\right)^{*}}$ is

$$
\mathbf{y}_{m}^{\left(f_{2}\right)^{*}}=-\mathbf{G}_{1(1)}^{\left(f_{2}\right)^{*}} d_{2}+\mathbf{G}_{1(2)}^{\left(f_{2}\right)^{*}} d_{1}+\mathbf{G}_{2}^{\left(f_{2}\right)^{*}} \mathbf{x}_{s}^{\left(f_{2}\right)^{*}}+\mathbf{n}_{m}^{\left(f_{2}\right)^{*}} .
$$

In order to deal with the interference coming from the small-cell system towards MUT, zero forcing is used. After the small-cell interference removal through the precoding done at the SBSs a standard Alamouti decoding process is performed, to detect the transmitted symbols at the MUT.

\subsubsection{Macro-Cell System with Double-SFBC Scheme}

As discussed before the SFBC scheme is restricted to 2 antennas at the transmitter side. Therefore, we consider

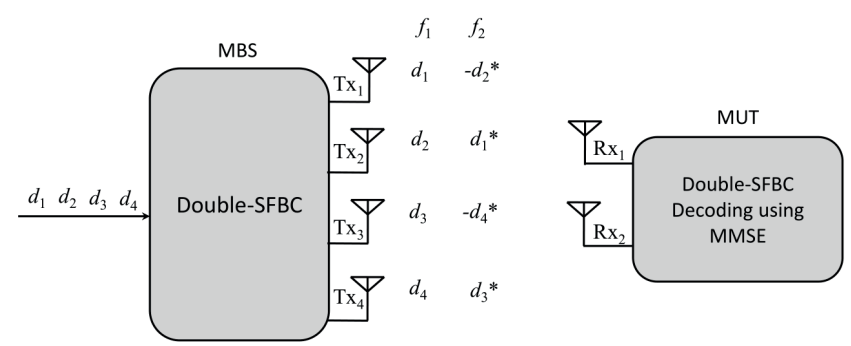

Fig. 4. Double-SFBC scheme at MBS.

${ }^{3}$ The OFDM based systems are usually designed so that channels between some adjacent carriers are approximately flat. the double-SFBC based scheme to be able to use more than 2 transmit antennas and increase the multiplexing gain. We assume that the transmitter has four $\left(M_{m}=4\right)$ and the receiver has a pair of antennas $\left(N_{m}=2\right)$, as shown in Fig. 4 . With this method two pairs of two data symbols are transmitted in parallel. The four data symbols are transmitted over four antennas on only two subcarriers $f_{1}$ and $f_{2}$ according to the following encoding matrix

$$
\mathbf{x}_{m}^{f_{1}}=\left[\begin{array}{l}
d_{1} \\
d_{2} \\
d_{3} \\
d_{4}
\end{array}\right], \mathbf{x}_{m}^{\left(f_{2}\right)^{*}}=\left[\begin{array}{c}
-d_{2} \\
d_{1} \\
-d_{4} \\
d_{3}
\end{array}\right] .
$$

For this case, the received signals at the $l^{\text {th }}$ antenna on frequencies $f_{1}$ and $f_{2}$ can be written as

$$
\begin{gathered}
\mathbf{y}_{m(l, 1)}^{f_{1}}=\mathbf{G}_{1(l, 1)}^{f_{1}} d_{1}+\mathbf{G}_{1(l, 2)}^{f_{1}} d_{2} \\
+\mathbf{G}_{1(l, 3)}^{f_{1}} d_{3}+\mathbf{G}_{1(l, 4)}^{f_{1}} d_{4}+\mathbf{G}_{2}^{f_{1}} \mathbf{x}_{s}^{f_{1}}+\mathbf{n}_{m}^{f_{1}}, \\
\mathbf{y}_{m(l, 1)}^{\left(f_{2}\right)^{*}}=-\mathbf{G}_{1(l, 1)}^{\left(f_{2}\right)^{*}} d_{2}+\mathbf{G}_{1(l, 2)}^{\left(f_{2}\right)^{*}} d_{1} \\
-\mathbf{G}_{1(l, 3)}^{\left(f_{2}\right)^{*}} d_{4}+\mathbf{G}_{1(l, 4)}^{\left(f_{2}\right)^{*}} d_{3}+\mathbf{G}_{2}^{\left(f_{2}\right)^{*}} \mathbf{x}_{s}^{\left(f_{2}\right)^{*}}+\mathbf{n}_{m}^{\left(f_{2}\right)^{*}} .
\end{gathered}
$$

Once again the interfering signal from the small-cell system at MUT, as seen in (9) and (10), is mitigated by using ZF. In this approach, after the small-cell interference removal the transmitted data symbols are separated by using a minimum mean square error (MMSE) based equalizer. In order to explain the decoding process to estimate the transmitted symbols at MUT, the combination of the four received signals at each antenna can be presented in matrix form as follows

$$
\begin{aligned}
{\left[\begin{array}{ll}
\mathbf{y}_{m(1,1)}^{f_{1}} & \mathbf{y}_{m(2,1)}^{f_{1}} \\
\mathbf{y}_{m(1,1)}^{f_{2}} & \mathbf{y}_{m(2,1)}^{f_{2}}
\end{array}\right] } & =\left[\begin{array}{cccc}
d_{1} & d_{2} & d_{3} & d_{4} \\
-d_{2}{ }^{*} & d_{1}^{*} & -d_{4}^{*} & d_{3}^{*}
\end{array}\right]\left[\begin{array}{ll}
\mathbf{G}_{1(1,1)}^{f_{1}} & \mathbf{G}_{1(2,1)}^{f_{1}} \\
\mathbf{G}_{1(1,2)}^{f_{1}} & \mathbf{G}_{1(2,2)}^{f_{1}} \\
\mathbf{G}_{1(1,3)}^{f_{1}} & \mathbf{G}_{1(2,3)}^{f_{1}} \\
\mathbf{G}_{1(1,4)}^{f_{1}} & \mathbf{G}_{1(2,4)}^{f_{1}}
\end{array}\right](11) \\
& +\left[\begin{array}{cc}
\mathbf{n}_{m(1,1)}^{f_{1}} & \mathbf{n}_{m(2,1)}^{f_{1}} \\
\mathbf{n}_{m(1,1)}^{f_{2}} & \mathbf{n}_{m(2,1)}^{f_{2}}
\end{array}\right]
\end{aligned}
$$

which is equivalent to have,

$$
\begin{aligned}
& {\left[\begin{array}{c}
\mathbf{y}_{m(1,1)}^{f_{1}} \\
\mathbf{y}_{m(2,1)}^{f_{1}} \\
\mathbf{y}_{m(1,1)}^{\left(f_{2}\right)^{*}} \\
\mathbf{y}_{m(2,1)}^{\left(f_{2}\right)^{*}}
\end{array}\right] }=\underbrace{\left[\begin{array}{llll}
\mathbf{G}_{1(1,1)}^{f_{1}} & \mathbf{G}_{1(1,2)}^{f_{1}} & \mathbf{G}_{1(1,3)}^{f_{1}} & \mathbf{G}_{1(1,4)}^{f_{1}} \\
\mathbf{G}_{1(2,1)}^{f_{1}} & \mathbf{G}_{1(2,2)}^{f_{1}} & \mathbf{G}_{1(2,3)}^{f_{1}} & \mathbf{G}_{1(2,4)}^{f_{1}} \\
\mathbf{G}_{1(1,2)}^{\left(f_{1}\right)^{*}} & -\mathbf{G}_{1(1,1)}^{\left(f_{1}\right)^{*}} & \mathbf{G}_{1(1,4)}^{\left(f_{1}\right)^{*}} & -\mathbf{G}_{1(1,3)}^{\left(f_{1}\right)^{*}} \\
\mathbf{G}_{1(2,2)}^{\left(f_{1}\right)^{*}} & -\mathbf{G}_{1(2,1)}^{\left(f_{1}\right)^{*}} & \mathbf{G}_{1(2,4)}^{\left(f_{1}\right)^{*}} & -\mathbf{G}_{1(2,3)}^{\left(f_{1}\right)^{*}}
\end{array}\right]}_{\mathbf{G}_{e q}}\left[\begin{array}{c}
d_{1} \\
d_{2} \\
d_{3} \\
d_{4}
\end{array}\right] \\
&+\left[\begin{array}{l}
\mathbf{n}_{m(1,1)}^{f_{1}} \\
\mathbf{n}_{m(2,1)}^{f_{1}} \\
\mathbf{n}_{m(1,1)}^{\left(f_{2}\right)^{*}} \\
\mathbf{n}_{m(2,1)}^{\left(f_{2}\right)^{*}}
\end{array}\right]
\end{aligned}
$$


Since we assume that $\mathbf{G}_{1}^{f_{1}} \approx \mathbf{G}_{1}^{f_{2}}$, we simplified the notation in (11) and (12) by using only the superscript $f_{1}$. It can be seen from (12) that the four transmitted data symbols can be separated by using MMSE equalizer $\left(\mathbf{w}_{M M S E}\right)$, which for this case is given by,

$$
\mathbf{w}_{M M S E}=\mathbf{G}_{e q}^{H}\left(\mathbf{G}_{e q} \mathbf{G}_{e q}^{H}+\sigma^{2} \mathbf{I}\right)^{-1} .
$$

\subsubsection{Small-Cell System}

The signal model of the small-cell system for the methods with SFBC scheme is the same as described in Sec. 2.1.2. The only difference is the number of subcarriers used at small-cells to detect the signals, for this case two subcarriers are used. The received signal $\left(\mathbf{y}_{s k}^{f_{1}}\right)$ at the $S U T_{k}$ on subcarrier $f_{1}$ is given by

$$
\mathbf{y}_{s k}^{f_{1}}=\mathbf{F}_{k}^{f_{1}} \mathbf{x}_{m}^{f_{1}}+\mathbf{H}_{k}^{f_{1}} \mathbf{x}_{s}^{f_{1}}+\mathbf{n}_{s k}^{f_{1}} .
$$

Similarly, at subcarrier $f_{2}$ after the application of the conjugate operator, the received signal $\mathbf{y}_{s k}^{\left(f_{2}\right)^{*}}$ at $S U T_{k}$ is

$$
\mathbf{y}_{s k}^{\left(f_{2}\right)^{*}}=\mathbf{F}_{k}^{\left(f_{2}\right)^{*}} \mathbf{x}_{m}^{f_{2}}+\mathbf{H}_{k}^{\left(f_{2}\right)^{*}} \mathbf{x}_{s}^{\left(f_{2}\right)^{*}}+\mathbf{n}_{s k}^{\left(f_{2}\right)^{*}} .
$$

To remove the interference generated by the MBS the $S U T_{k}$ applies the filter matrix $\mathbf{W}_{k}^{f}$ to the concatenation of the received signals at subcarriers $f_{1}$ and $f_{2} \quad\left(\mathbf{y}_{s k}^{f}\right.$ $\left.=\left[\left(\mathbf{y}_{s k}^{f_{1}}\right)^{H},\left(\mathbf{y}_{s k}^{\left(f_{2}\right)^{*}}\right)^{H}\right]^{H}\right)$

$$
\mathbf{z}_{s k}^{f}=\mathbf{W}_{k}^{f} \mathbf{y}_{s k}^{f} .
$$

The filter matrix $\mathbf{W}_{k}^{f}$ is designed in the next sections, by forcing to zero the interference arriving from the MBS.

\section{Precoders and Filter Matrices Design}

We design the precoder and filter matrices of the macro-cell and small-cell terminals, such that the two systems can coexist within the same spectrum. We consider different levels of cooperation between the two systems in order to design the filter matrix of our proposed schemes. First, we summarize the previously proposed methods of [28]. Then, we present in detail the proposed coordinated $2 n$-Bit and the joint IA with SFBC and double-SFBC schemes.

\subsection{Extended and Proposed Methods without SFBC}

In this section, first we extend the methods discussed in [28], where we considered a specific scenario with each terminal having two antennas except the macro UT that has a single antenna. In this work, we extend those schemes for a general number of antennas at each terminal and for the case where OFDM modulation is considered. Then we design a new IA based scheme for the considered hetero- geneous systems. Namely, the coordinated $2 n$-Bit approach, which is an extension of the 2-bit method proposed in [28].

\subsubsection{Full-Coordinated Method}

Assuming the knowledge of the $\mathbf{G}_{1}^{f}$ channel at the MBS, the data vector can be linear precoded to efficiently separate the spatial streams at the MUT. For the case where the MUT is equipped with single antenna a maximal ratio transmission (MRT) based precoder can be employed as in [28]. When the MUT is equipped with an antenna array a ZF or MMSE based precoders can be used. In this work, we consider the ZF based precoder at the MBS given by

$$
\mathbf{V}_{m}^{f}=\mathbf{G}_{1}^{f^{H}}\left(\mathbf{G}_{1}^{f} \mathbf{G}_{1}^{f^{H}}\right)^{-1} .
$$

It is assumed that the macro-cell system is not aware of the presence of small-cell system within its boundaries and the precoder $\mathbf{V}_{m}^{f}$ used at the macro-cell system does not change due to the existence of small-cell terminals. In the downlink the MBS interferes with the SUTs. From (1) and (4), we can see that to enforce the zero interference condition and mitigate the interference coming from MBS the filter matrix at $S U T_{k}$ must satisfy

$$
\mathbf{W}_{k}^{f} \mathbf{F}_{k}^{f} \mathbf{V}_{m}^{f}=0 .
$$

Let us define matrix $\mathbf{A}^{f}=\operatorname{null}\left(\mathbf{V}_{m}^{f}\right)$, which we denominate by alignment direction in the following, then from (18) we have

$$
\mathbf{W}_{k}^{f} \mathbf{F}_{k}^{f}=\mathbf{A}^{f} .
$$

From the point of view of each $S U T_{k}, k=\{1, \ldots, K\}$ the equivalent channel $\mathbf{W}_{k}^{f} \mathbf{F}_{k}^{f}$ is identical to $\mathbf{A}^{f}$. That is, the filter matrix aligns all channels and the final channel is orthogonal to the MBS precoder so that the zero interference condition is satisfied.

From (18) it follows that to satisfy the zero interference condition the filter matrix $\left(\mathbf{W}_{k}^{f}\right)$ at SUTs is

$$
\begin{aligned}
\mathbf{W}_{k}^{f} & =\operatorname{null}\left(\mathbf{F}_{k}^{f} \mathbf{V}_{m}^{f}\right), \\
\mathbf{A}^{f} & =\operatorname{null}\left(\mathbf{V}_{m}^{f}\right) .
\end{aligned}
$$

where $\mathbf{A}^{f}$ is the alignment direction that completely describes the MBS interfering signal towards the SUTs. Assuming the knowledge of alignment direction or equivalently of the MBS precoder the small-cells can align their transmission accordingly without experiencing any interference from macro-cell system.

As can be seen from (20) and (21), the design of matrix $\mathbf{W}_{k}^{f}$ requires the knowledge of the precoder $\mathbf{V}_{m}^{f}$ and channels $\mathbf{F}_{k}^{f}$. The channels $\mathbf{F}_{k}^{f}$ as described in Sec. 2.1.2, are quasi-static and they can be easily estimated at the SUTs. On the other hand, the precoder $\mathbf{V}_{m}^{f}$ changes on every TTI since it depends on the channel between the 
MBS and MUT, i.e. $\mathbf{G}_{1}^{f}$. This means that alignment direction matrix must be exchanged between the two systems every TTI. Other possible strategy consists in estimating the equivalent channel $\mathbf{F}_{k}^{f} \mathbf{V}_{m}^{f}$, by listening to the pilot signals, but it will also require a high pilot density.

Let us now discuss the interference that SBSs may cause in the MUT. Evidently, the small-cell system should not interfere with the macro-cell system (i.e. the macro-cell has the priority to access the available resources). Also the SUTs should not interfere with each other. We consider that the SBSs are connected via backhaul network to a CU in order to perform joint processing of transmitted signals. The CU has enough DoF (i.e. $K M_{s}$ ) to cancel both the interference that the SBSs cause in the MUT and the interference between SUTs. In order to design the precoding matrix at the $\mathrm{CU} \mathrm{ZF}$ is used, to zero force the macro-cell and small-cell channels together. In this context, the $\mathrm{ZF}$ precoder $\mathbf{V}_{s}^{f}$, computed at the $\mathrm{CU}$, is given by

$$
\mathbf{V}_{s}^{f}=\mathbf{B}^{f^{H}}\left(\mathbf{B}^{f} \mathbf{B}^{f^{H}}\right)^{-1}
$$

where $\quad \mathbf{B}^{f}=\mathbf{W}^{f} \mathbf{H}_{e q}^{f}, \quad \mathbf{H}_{e q}^{f}=\left[\left(\mathbf{G}_{2}^{f}\right)^{H},\left(\mathbf{H}_{1}^{f}\right)^{H}, \ldots,\left(\mathbf{H}_{K}^{f}\right)^{H}\right]^{H}$ and $\mathbf{W}^{f}=\operatorname{diag}\left(\mathbf{I}, \mathbf{W}_{1}^{f}, \ldots \mathbf{W}_{k}^{f}, \ldots \mathbf{W}_{K}^{f}\right)$. The filter matrix $\mathbf{W}_{k}^{f}$ is known at the CU since the channels $\mathbf{F}_{k}^{f}$ are quasistatic, the SUTs may feedback them to the CU without much overhead requirements.

\subsubsection{Uncoordinated-Static Method}

For this method we follow the same procedure to remove the interferences (inter-system and between smallcell UTs) as the previous one, but now we consider that the precoder at MBS is static at the beginning of interaction between the two systems, i.e. its value does not change every TTI. As the precoder $\mathbf{V}_{m}^{f}$ is static, it is assumed that its value is also known at the small-cell terminals. Therefore, this method requires no inter-system cooperation. For example we assume the precoder at MBS is the all ones matrix, i.e. $\mathbf{V}_{m}^{f}=\mathbf{1}$.

\subsubsection{Coordinated 2n-Bit Method}

In order to achieve a commitment between performance and inter-system information-exchange requirements of the full-coordinated and uncoordinated-static methods, we propose a coordinated $2 n$-bit method. For this case we consider the same precoder used for full-coordinated approach in order to design the alignment direction. However, only a quantized version of the alignment vector is exchanged between the two systems. Therefore, we quantize the alignment direction with $2 n$ bits ( $n$ bits for the real and $n$ bits for the complex part, where $n=1,2,3, .$.$) . The$ quantized alignment direction is given by

$$
\mathbf{A}_{q}^{f}=f_{Q}\left(\operatorname{Re}\left\{\left(\mathbf{A}^{f}\right)\right\}\right)+j f_{Q}\left(\operatorname{Im}\left\{\left(\mathbf{A}^{f}\right)\right\}\right)
$$

where $f_{Q}($.$) denotes a quantization function, the R e\{$.$\} and$ $\operatorname{Im}\{$.$\} are the real and imaginary parts of alignment direc-$ tion $\mathbf{A}^{f}$. In this manuscript, for the sake of simplicity, we consider only uniform quantizers. To remove both the interference from the MBS to the SUTs and the interference that the SBSs cause in the MUT we follow the same procedure as for the full coordinated method. Notice that for this case the MBS precoder is also quantized, by taking into account the zero interference condition $\mathbf{A}_{q}^{f}=\operatorname{null}\left(\mathbf{V}_{m, q}^{f}\right), \mathbf{V}_{m, q}^{f}$ is a quantized version of $\mathbf{V}_{m}^{f}$.

\subsection{Joint IA with SFBC/Double-SFBC Methods}

In this section, with the aim to completely eliminate the need to exchange any information between the macro and small-cell systems, we design new joint IA and SFBC/double-SFBC schemes.

As seen in the full-coordinated and coordinated $2 n$ Bit methods, the design of the precoder at the MBS and filter matrix at the SUTs depends on the channel $\mathbf{G}_{1}^{f}$ between the MBS and MUT. The key idea behind the use of $\mathrm{SFBC} /$ double-SFBC at the macro-cell system is that, it allows the design of filter matrix at SUTs without having any coordination between the two systems. More specifically, the small-cells just need to sense that the macro-cell system is using a SFBC/double-SFBC scheme.

Lets start by describing both the design of the alignment direction and filter matrix for the methods with SFBC (i.e. joint IA with SFBC and joint IA with Double-SFBC). For the sake of simple representation, we can rewrite the received signals in equations (14) and (15), $\mathbf{y}_{s k}^{f_{1}}$ and $\mathbf{y}_{s k}^{\left(f_{2}\right)^{*}}$ (after the application of the conjugate operator) at the $S U T_{k}$ on subcarrier $f_{1}$ and $f_{2}$ in matrix form as follows

$$
\begin{aligned}
{\left[\begin{array}{c}
\mathbf{y}_{s k}^{f_{1}} \\
\mathbf{y}_{s k}^{\left(f_{2}\right)^{*}}
\end{array}\right] } & =\left[\begin{array}{cc}
\mathbf{F}_{k}^{f_{1}} & 0 \\
0 & \mathbf{F}_{k}^{\left(f_{2}\right)^{*}}
\end{array}\right]\left[\begin{array}{c}
\mathbf{x}_{m}^{f_{1}} \\
\mathbf{x}_{m}^{\left(f_{2}\right)^{*}}
\end{array}\right]+\left[\begin{array}{cc}
\mathbf{H}_{k}^{f_{1}} & 0 \\
0 & \mathbf{H}_{k}^{\left(f_{2}\right)^{*}}
\end{array}\right]\left[\begin{array}{c}
\mathbf{x}_{s}^{f_{1}} \\
\mathbf{x}_{s}^{\left(f_{2}\right)^{*}}
\end{array}\right] \\
& +\left[\begin{array}{c}
\mathbf{n}_{s k}^{f_{1}} \\
\mathbf{n}_{s k}^{\left(f_{2}\right)^{*}}
\end{array}\right]
\end{aligned}
$$

According to (5) for the Alamouti SFBC and (8) for the double-Alamouti SFBC the concatenation of the transmitted signal on subcarriers $f_{1}$ and $f_{2}$ is given, respectively, by

$$
\left[\begin{array}{c}
\mathbf{x}_{m}^{f_{1}} \\
\mathbf{x}_{m}^{\left(f_{2}\right)^{*}}
\end{array}\right]=\underbrace{\left[\begin{array}{cc}
1 & 0 \\
0 & 1 \\
0 & -1 \\
1 & 0
\end{array}\right]}_{\mathbf{V}_{m}^{f, 2}} \underbrace{\left[\begin{array}{c}
d_{1} \\
d_{2}
\end{array}\right]}_{\mathbf{d}_{m}^{f_{1}, 2}}=\mathbf{V}_{m}^{f_{1,2}} \mathbf{d}_{m}^{f_{1,2}}
$$




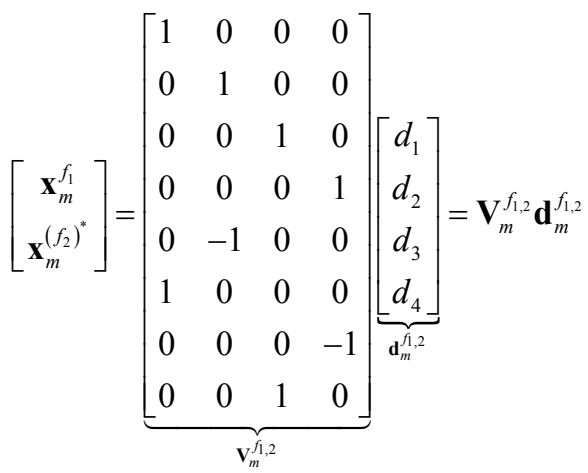

The MBS precoder $\mathbf{V}_{m}^{f_{1,2}}=\left[\mathbf{V}_{m}^{\left(\mathrm{f}_{1}\right)^{H}}, \mathbf{V}_{m}^{\left(\mathrm{f}_{2}\right)^{H}}\right]^{H}$ for this case is applied jointly for two consecutive subcarriers $f_{1}$ and $f_{2}$. Notice that for this case the precoder is constant and independent of the macro channel $\mathbf{G}_{1}^{f}$, i.e. $\mathbf{V}_{m}^{f_{1,2}} \in\{-1,0,1\}^{2 M_{m} \times 2 N_{m}}$.

In order to cancel the interference coming from MBS towards the $S U T_{k}$, we need to compute an appropriate filter matrix at the $S U T_{k}$. Let the filter matrix $\mathbf{W}_{k}^{f_{1,2}}$ based on equation (20) for the two subcarriers be given by

$$
\mathbf{W}_{k}^{f_{1,2}}=\operatorname{null}\left(\mathbf{F}_{k}^{f_{1,2}} \mathbf{V}_{m}^{f_{1,2}}\right)
$$

where $\mathbf{F}_{k}^{f_{1,2}}=\operatorname{diag}\left(\mathbf{F}_{k}^{f_{1}}, \mathbf{F}_{k}^{\left(f_{2}\right)^{*}}\right)$ and $\mathbf{V}_{m}^{f_{1,2}}$ denotes the constant matrices shown in (5) and (8) for the Alamouti and double-Alamouti SFBCs, respectively. As $\mathbf{V}_{m}^{f_{1,2}}$ is a constant matrix for these two cases, there is no need to exchange any information from the macro-cell to the small cells for the computation of the filtering matrices, contrarily to the full coordinated and coordinated $2 n$-Bit methods.

The alignment direction $\mathbf{A}^{f_{1,2}}$ for both joint IA with SFBC and joint IA with Double-SFBC schemes spans two subcarriers and is expressed, respectively as

For the joint IA with SFBC method:

$$
\mathbf{A}^{f_{1,2}}=\left[\begin{array}{cccc}
1 & 0 & 0 & -1 \\
0 & 1 & 1 & 0
\end{array}\right]
$$

For the joint IA with Double-SFBC method:

$$
\mathbf{A}^{f_{1,2}}=\left[\begin{array}{cccccccc}
1 & 0 & 0 & 0 & 0 & -1 & 0 & 0 \\
0 & 1 & 0 & 0 & 1 & 0 & 0 & 0 \\
0 & 0 & 1 & 0 & 0 & 0 & 0 & -1 \\
0 & 0 & 0 & 1 & 0 & 0 & 1 & 0
\end{array}\right]
$$

From (25) and (26) we verify that by setting the alignment as described in equations (28) and (29) for the joint IA with SFBC and joint IA with Double-SFBC methods respectively, the interference from MBS is completely removed at SUTs. This is made possible due to the redundancy present in the MBS transmitted data symbols.

\subsection{Information Exchange Requirements Comparison}

The full-coordinated method has the highest intersystem information sharing requirements, since the macrocell system must coordinate with the small-cell system in order to share $2 M_{m} N_{m}$ real numbers, per subcarrier. For an OFDM based system $2 M_{m} N_{m} N_{c}$ reals can be huge number. The uncoordinated-static approach does not require any information exchange between the two systems, but as shown in the next section, this results in significant performance degradation for the macro-cell system.

To achieve a good trade-off between information exchange overhead and performance, we propose a coordinated $2 n$-Bit method that enables a significant reduction in the information exchange requirements, i.e. from $2 M_{m} N_{m} N_{c}$ real numbers to $2 n M_{m} N_{m} N_{c}$ bits. This method, as shown in Sec. 4, achieves close to optimal performance.

The specification for methods that can achieve performance close to the full-coordinated method without any information exchange is of great importance. Therefore, we also propose a new joint IA with $\mathrm{SFBC} /$ double-SFBC scheme where no information needs to be exchanged between the two systems, i.e. these approaches have the same requirements as the uncoordinated static approach, but with a performance close to the full-coordinated method. A summary of the inter-system information-exchange requirements is presented in Tab. 1 .

\begin{tabular}{|c|c|}
\hline Methods & $\begin{array}{c}\text { Information-Exchange } \\
\text { Requirements }\end{array}$ \\
\hline Full-Coordinated & $2 M_{m} N_{m} N_{c}$ Real Number \\
\hline Uncoordinated-Static & 0 \\
\hline Coordinated 2n-Bit & $2 n M_{m} N_{m} N_{c}$ bits \\
\hline Joint IA and SFBC & 0 \\
\hline Joint IA with Double-SFBC & 0 \\
\hline
\end{tabular}

Tab. 1. Comparison of inter-system information exchange requirements.

\section{Simulation Results and Discussion}

In this section, we provide the performance assessment of our proposed methods. We compare them to the full-coordinated and uncoordinated-static methods with the help of simulations. We consider scenarios, with 2 smallcells (i.e. $K=2$ ) within the coverage area of a MBS but adding more small-cells will not impact on the performance of macro-cell system, since the interference can be completely eliminated irrespective the number of smallcells. We assume that the SBSs are able to cooperate through a backhaul network to a CU to perform joint processing of signals. We consider two scenarios: 
- Scenario 1: The number of antennas at the MBS, SBSs and SUTs is 2, and single antenna MUT, i.e. $M_{m}=M_{s}=N_{s}=2, N_{m}=1$

- Scenario 2: The number of antennas at the MBS, SBSs and SUTs is 4, and 2 at the MUT $M_{m}=M_{s}=N_{s}=4, N_{m}=2$.

It is assumed that SBSs are uniformly distributed within the boundaries of a macro-cell. The coverage radius of the MBS and each SBS is $2000 \mathrm{~m}$ and $600 \mathrm{~m}$, respectively and the power of the MUT is assumed to be 4 times higher than the power of SUTs.

We consider the ITU pedestrian channel model B, with modified tap delays according to the sampling frequency specified in LTE standards. A path loss exponent of 3.5 has been considered. The SNR at the cell edge is defined as $\left(P_{t} R^{-\theta} / \sigma^{2}\right)$, where $\theta$ is the path loss exponent and $R$ is the cell-radius, where it can be equal to $600 \mathrm{~m}$ or $2000 \mathrm{~m}$ for macro-cell and small-cell systems, respectively. $P_{t}$ is the transmit power at UT and it can be equal to $P_{m}$, if the Bit-Error Rate (BER) is measured for the macro-cell system or it can be equal to $P_{s}$, if the BER is calculated for the small-cell system. The OFDM parameters used for simulating both the macro-cell and small-cell systems are: FFT size $=1024$ (where only 128 subcarriers are used for both the systems); sampling frequency $f_{s}=15.36 \mathrm{MHz}$; cyclic prefix length $c_{p}=5.21 \mu \mathrm{s}$ and subcarrier separation is $15 \mathrm{kHz}$. In order to allow an appropriate comparison, all the considered methods are evaluated for the same spectral efficiency. Therefore, we used 16-QAM modulation for the proposed joint IA with SFBC, the coordinated $2 n$-bit, fullcoordinated and uncoordinated-static schemes, and QPSK for the proposed IA with double-SFBC one.

Let us start by considering the first scenario. For this case we compare the performance of the coordinated $2 n$-bit, joint IA with SFBC, full-coordinated, and uncoordinated-static methods. In Fig. 5 and Fig. 6, we present the BER performance of the first scenario (using 16-QAM modulation for all the curves) for the macro-cell and smallcell systems. In Fig. 5, we compare the performance of the proposed coordinated $2 n$-bit method by using different values of $n=1,2,3$ with the full-coordinated and uncoordinated-static methods.

As it can be verified from Fig. 5, the performance of coordinated 4-bit and 6-bit methods almost overlaps with full-coordinated method. On the other hand, the performance of coordinated 2-bit approach is also quite close to the optimal performance. As the performance of the 2-bit is very close to the performance of the 4 and 6-bit cases the 2-bit is preferable as the information exchange requirements are lower. The BER performance of the joint IA with SFBC approach has a gap of around $3 \mathrm{~dB}$ as compared to the full-coordinated case, since SFBC scheme can provide an array gain of 1 . On the other hand, the information-exchange requirements of joint IA with SFBC and uncoordinated-static methods are similar but the joint IA

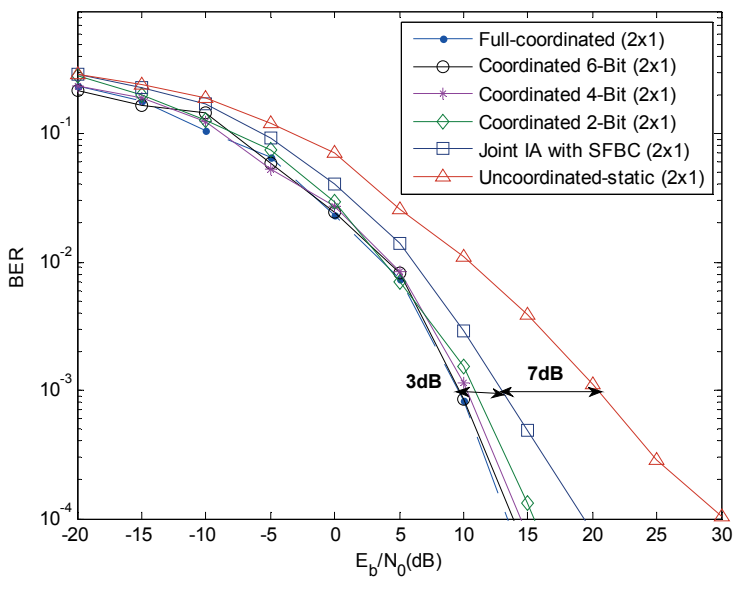

Fig. 5. BER performance for the macro-cell system and for scenario 1.

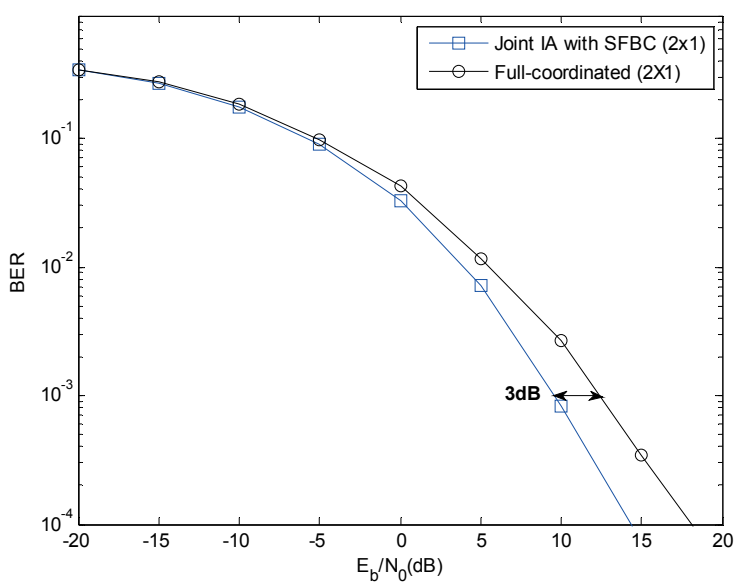

Fig. 6. BER performance for the small-cell system and for scenario 1 .

with SFBC scheme provides much better performance (a gap or around $7 \mathrm{~dB}$ for a target BER of $10^{-3}$ ) as compared to the uncoordinated-static method.

The BER curve of the small-cell system (for the first scenario) is presented in Fig. 6. We just compared the fullcoordinated and joint IA with SFBC methods since the performance of full-coordinated, coordinated $2 n$-bit and uncoordinated-static methods are identical. This can be explained by the fact that the design of filter matrix is not dependent on the small-cell channels $\left[\mathbf{H}_{k}^{f}\right]_{1 \leq k \leq K}$. Therefore, the equivalent channel preserves the original channel distribution. As verified from Fig. 6, the BER performance of joint IA with SFBC has around $3 \mathrm{~dB}$ better performance as compared to the full-coordinated approach. This is due to the fact that for the SFBC scheme every symbol is transmitted over two subcarriers, contrarily to the full-coordinated method where each symbol only spans one subcarrier.

Let us now consider the second scenario. For this case we compare the performance of the coordinated $2 n$-bit, joint IA with Double-SFBC, full-coordinated, and uncoordinated-static methods. In Fig. 7 and Fig. 8, we present the BER performance of our second scenario for the macro- 
cell and small-cell system, respectively. Notice that in this scenario we used QPSK modulation for the joint IA with Double-SFBC approach. From Fig. 7, we can observe that the coordinated $2 n$-Bit approach once again provides quite close to optimal performance. On the other hand, the performance of joint IA with Double-SFBC approach has a gap of around $3 \mathrm{~dB}$ as compared to the full-coordinated method and achieves much better performance (a gap of around $12 \mathrm{~dB}$ for a target BER of $10^{-3}$ ) as compared to the uncoordinated-static scheme, even if the information-exchange requirements of both the schemes are identical. In Fig. 8, we compare the BER performance of proposed joint IA with Double-SFBC with the full-coordinated method for the small-cell system. The proposed joint IA with Double-SFBC scheme provides around $3 \mathrm{~dB}$ better performance as compared to the case where full coordination is allowed between the two tiers.

In Fig. 9 and Fig. 10, we compare the BER curves of joint IA with Double-SFBC (using QPSK modulation) and joint IA with SFBC (using 16-QAM modulation) schemes for the macro-cell and small-cell system, respectively.

As it can be seen from Fig. 9, the performance of joint IA with Double-SFBC is around $5 \mathrm{~dB}$ better as compared

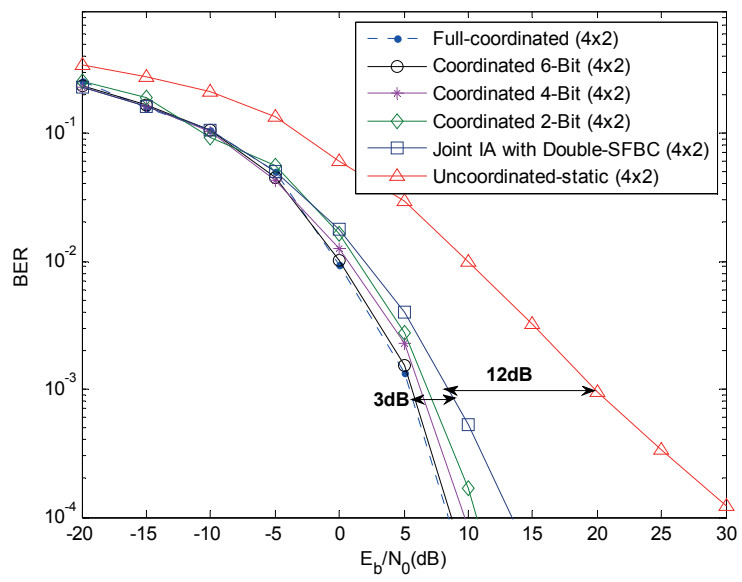

Fig. 7. BER performance for the macro-cell system and for scenario 2.

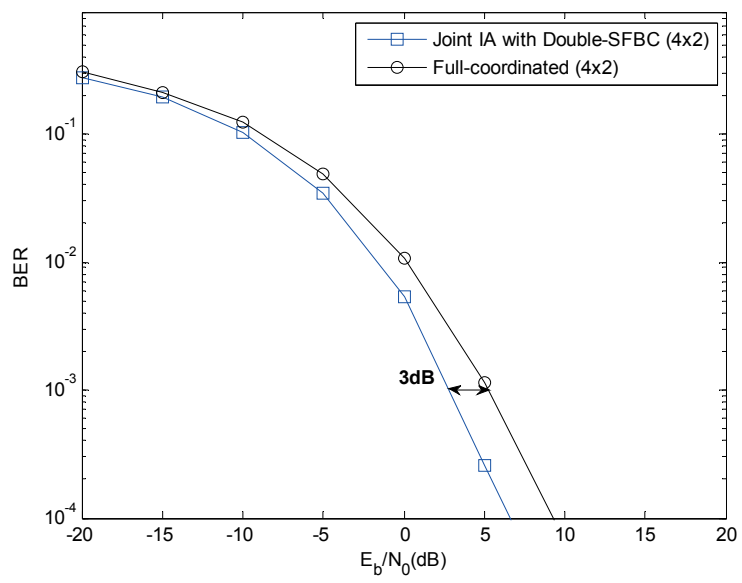

Fig. 8. BER performance for the small-cell system and for scenario 2 .

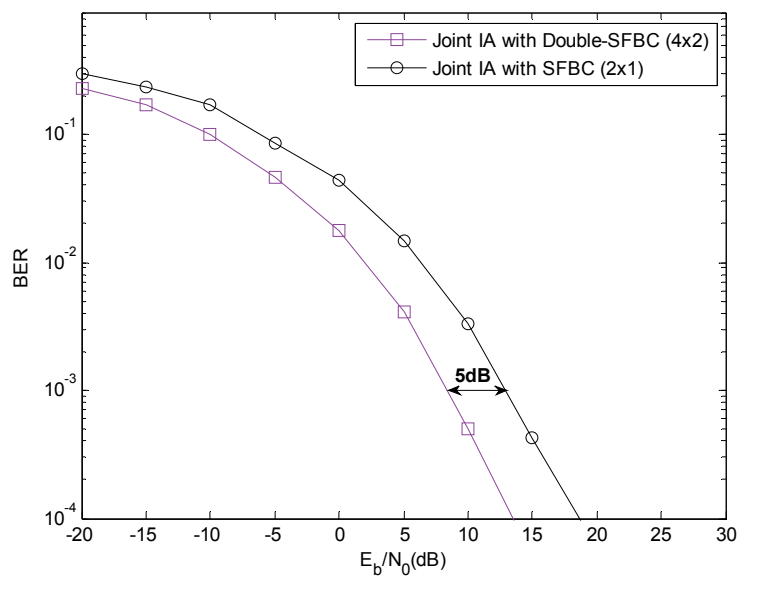

Fig. 9. BER performance at macro-cell system for Joint IA with Double-SFBC and Joint IA with SFBC.

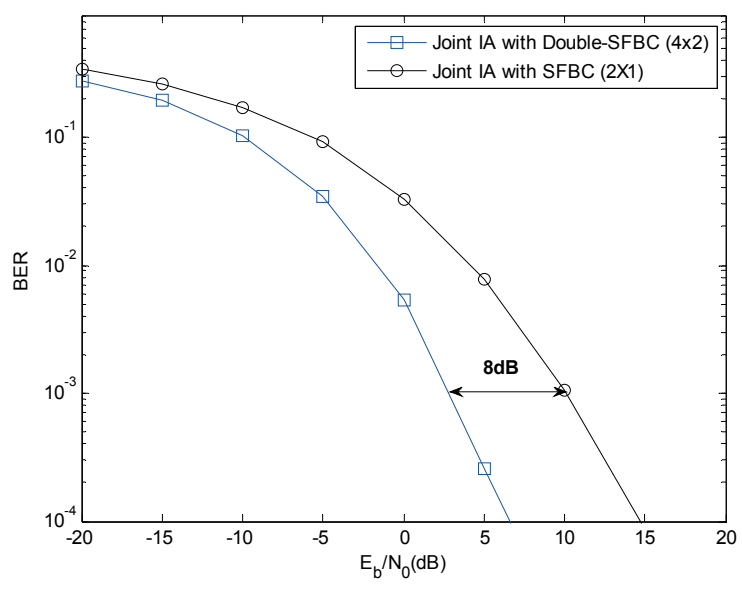

Fig. 10. BER performance at small-cell system for Joint IA with Double-SFBC and Joint IA with SFBC.

to the case where standard SFBC scheme is used in conjunction with IA for the macro-cell system. At the smallcell system, the performance of joint IA with DoubleSFBC is around $8 \mathrm{~dB}$ better than the joint IA with SFBC scheme as shown in Fig. 10. This is due to the fact that the Double-SFBC scheme offers both the transmit and receive diversity as compared to the standard SFBC scheme where it provides just the transmit diversity. Moreover, high order modulation (16-QAM) is used for the joint IA with SFBC and therefore it is more prone to errors than the IA with double-SFBC that uses QPSK modulation.

\section{Conclusion}

In this paper, we proposed several interference alignment schemes for the downlink of heterogeneous based systems, where a set of small-cells coexists with the macrocell sharing the same spectrum. The full-coordinated method provides the best performance but with very high inter-system information-exchange requirements. On the other hand, for the uncoordinated-static approach, there is no need of information exchange between the two systems but the macro-cell system experiences the worst performance, which is not acceptable for the macro-cell system, 
since it has the priority to access the available resources. To overcome the shortcomings of full-coordinated and the uncoordinated-static methods, we proposed the coordinated $2 n$-bit and joint IA with SFBC/double-SFBC schemes. We have shown that using only $2-4$ bit to quantize the alignment direction is enough to achieve a performance close to the one given by the full-coordinated approach.

The proposed joint IA with SFBC/Double-SFBC schemes enables the small-cell system to opportunistically access the free space resources of the macro-cell system without degrading its performance. Moreover, these methods provide improved performance with comparable inter-system information-exchange requirements to the uncoordinated-static approach. Thus, the proposed methods allow the network to achieve the benefits of full-coordinated and uncoordinated-static methods without their main drawbacks. To conclude we can clearly state that the proposed methods can be very useful for the future $5 \mathrm{G}$ based networks.

\section{Acknowledgments}

This work was supported by the Portuguese Fundação para a Ciência e Tecnologia (FCT) COPWIN (PTDC/EEITEL/1417/2012), ADIN (PTDC/EEI-TEL/2990/2012) and HETCOP (PEst-OE/EEI/LA0008/2013) projects and FCT grant for the first (SFRH/BD/94548/2013) and second (SFRH/BPD/95375/2013) authors.

\section{References}

[1] LI, Q., NIU, H., PAPATHANASSIOU, A., WU, G. 5G Network capacity- key elements and technologies. IEEE Vehicular Technology Magazine, 2014, vol. 9, no. 1, p. 71-78. DOI: 10.1109/MVT.2013.2295070

[2] DAMNJANOVIC, A. A survey on 3GPP heterogeneous networks. IEEE Wireless Communication Magazine, 2011, vol. 18, no. 3, p. 10-21. DOI: 10.1109/MWC.2011.5876496

[3] NAKAMURA, T., NAGATA, S., BENJEBBOUR, A., KISHIYAMA, Y., TANG HAI, SHEN XIAODONG, YANG NING, LI NAN. Trends in small cell enhancements in LTE advanced. IEEE Communication Magazine, 2013, vol. 51, no. 2, p. 98-105. DOI: 10.1109/MCOM.2013.6461192

[4] HOADLEY, J., MAVEDDAT, P. Enabling small cell deployment with HetNet. IEEE Wireless Communication Magazine, 2013, vol. 19, no. 2, p. 4-5. DOI: 10.1109/MWC.2012.6189405

[5] YONG, S. S., QUEK, T. Q. S., KOUNTOURIS, M., HYUNDONG, S. Energy efficient heterogeneous cellular networks. IEEE Journal on Selected Areas in Communications, 2013, vol. 31, no. 5, p. 840-850. DOI: 10.1109/JSAC.2013.130503

[6] CHANDRASEKHAR, V., ANDREWS, J. 5G on the Horizon: Key challenges for the radio-access network. IEEE Vehicular Technology Magazine, 2014, vol. 8, no. 3, p. 47-53. DOI: 10.1109/MVT.2013.2269187

[7] VALENTA, V., MARSALEK, R., BAUDOIN, G., VILlEGAS, M., SUAREZ, M., ROBERT, F. Survey on spectrum utilization in
Europe: measurements, analyses and observations. In Proceedings of the IEEE Cognitive Radio Oriented Wireless Networks Communications. Cannes (France), 2010, 5p.

[8] CHENG, S. M., LIEN, S. Y., CHIU, F. S, CHEN, K. C. On exploiting cognitive radio to mitigate interference in macro/femto heterogeneous networks. IEEE Wireless Communication Magazine, 2011, vol. 18, no. 3, p. 40-47. DOI: 10.1109/MWC.2011.5876499

[9] ZAHIR, T., ARSHAD, K., NAKATA, A., MOESSNER, K. Interference management in femtocells. IEEE Communications Surveys and Tutorials, 2013, vol. 15, no. 1, p. 293-311. DOI: 10.1109/SURV.2012.020212.00101

[10] SAQUIB, N., HOSSAIN, E., LONG BAO LE, DONG IN KIM. Interference management in OFDMA femtocell networks: issues and approaches. IEEE Wireless Communications, 2012, vol. 19, no. 3, p. 86-95. DOI: 10.1109/MWC.2012.6231163

[11] SRINIVASA, S., JAFAR, S. A. Cognitive radios for dynamic spectrum access - The throughput potential of cognitive radio: A theoretical perspective. IEEE Communication Magazine, 2007, vol. 45, no. 5, p. 73-79. DOI: 10.1109/MCOM.2007.358852

[12] LU LU, XINGWEI ZHOU, UZOMA ONUNKWO, GEOFFREY YE-LI. Ten years of research in spectrum sensing and sharing in cognitive radio. EURASIP Journal on Wireless Communications and Networking, 2012, vol. 28. DOI: 10.1186/1687-1499-2012-28

[13] MUSHTAQ, M. T., KHAN, I., KHAN, M. S., KOUDELKA, O. Signal detection for QPSK based cognitive radio systems using support vector machines. Radioengineering, April 2015, vol. 24, no. 1, p. 192-198. DOI: 10.13164/re.2015.0192

[14] GOLDSMITH, A., JAFAR, S., MARIC, I., SRINIVASA, S Breaking spectrum gridlock with cognitive radios: An information theoretic perspective. In Proceedings of the IEEE, 2009, vol. 97, no. 5, p. 894-914. DOI: 10.1109/JPROC.2009.2015717

[15] BHARUCHA, Z., HAAS, H., AUER, G., COSOVIC, I. Femto cell resource partitioning. In Proceedings of the IEEE GLOBECOM Workshops. Honolulu (USA), 2009, 6 p. DOI: 10.1109/GLOCOMW.2009.5360723

[16] GORA, J., PEDERSEN, K. I., SZUFARSKA, A., STRZYZ, S Cell-specific uplink power control for heterogeneous networks in LTE. In Proceedings of the IEEE, Vehicular Technology Conference Fall (VTC). Ottawa (Canada), 2010, 5 p. DOI: 10.1109/VETECF.2010.5594439

[17] YUANYE, W., PEDERSEN, K. I. Performance analysis of enhanced inter-cell interference coordination in LTE-advanced heterogeneous networks. In Proceedings of the IEEE Vehicular Technology Conference Spring (VTC). Yokohama (Japan), 2012, 5 p. DOI: 10.1109/VETECS.2012.6240233

[18] MATINMIKKO, M., MUSTONEN, M., HOYHTYA, M., RAUMA, T., SARVANKO, H., MAMMELA, A. Distributed and directional spectrum occupancy measurements in the $2.4 \mathrm{GHz}$ ISM band. In Proceedings of the IEEE 7th International Symposium on Wireless Communication Systems (ISWCS). York (UK), 2010, p. 676-980. DOI: 10.1109/ISWCS.2010.5624366

[19] ATTAR, A., KRISHAMURTHY, V., GHAREHSHIRAN, O. N Interference management using cognitive base-stations for UMTS LTE. IEEE Communication Magazine, 2011, vol. 49, no. 8, p. 152 to 159. DOI: 10.1109/MCOM.2011.5978429

[20] YAACOUB, E., DAWY, Z. Enhancing the performance of OFDMA underlay cognitive radio networks via secondary pattern nulling and primary beam steering. In Proceedings of the IEEE Wireless Communications and Networking Conference (WCNC). Cancun, Quintana Roo (Mexico), 2011, p. 1476-1481. DOI: 10.1109/WCNC.2011.5779348

[21] CADAMBE, V., JAFAR, S. Interference alignment and degrees of freedom of the k-user interference channel. IEEE Transactions on 
Information Theory, 2009, vol. 54, no. 8, p. 3425-3441. DOI: 10.1109/TIT.2009.2035244

[22] SUH, C., HO, M., TSE, D. Downlink interference alignment. IEEE Transactions on Communications, 2011, vol. 59, no. 9, p. 2616 to 2626. DOI: 10.1109/TCOMM.2011.070511.100313

[23] SHIN, W., LEE, N., NOH, W., CHOI, H-HO., CLERCKX, B., SHIN, C., JANG, K. Hierarchical interference alignment for heterogeneous networks with multiple antennas. In Proceedings of the IEEE International Communications Workshops (ICC). Kyoto (Japan), 2011, 6 p. DOI: 10.1109/iccw.2011.5963547

[24] MASO, M., CARDOSO, L. S., DEBBAH, M., VANGELISTA, L. Cognitive interference alignment for OFDM two-tired networks. In Proceedings of the IEEE 13th International Workshop on Signal Processing Advances in Wireless Communications (SPAWC). Cesme (Turkey), 2012, p. 244-248. DOI: 10.1109/SPAWC.2012.6292902

[25] GULER, B., YENER, A. Selective interference alignment for MIMO cognitive femtocell networks. IEEE Journal on Selected Areas in Communications, 2014, vol. 32, no. 3, p. 439-450. DOI: 10.1109/JSAC.2014.140306

[26] SHARMA, S., CHATZINOTAS, S., OTTERSTEN, B. Interference alignment for spectral coexistence of heterogeneous networks. EURASIP Journal on Wireless Communications and Networking, 2013. DOI: 10.1186/1687-1499-2013-46.

[27] CASTANHEIRA, D., GAMEIRO, A., SILVA, A. One-bit nullspace cognitive interference alignment for heterogeneous networks. In Proceedings of the IEEE 24th International Symposium on Personal Indoor and Mobile Radio Communications. London (UK), 2013, p. 1431-1435. DOI: 10.1109/PIMRC.2013.6666366.

[28] Ali, S. S., CAStanheira, D., Silva, A., Gameiro, A. Downlink cognitive interference alignment for heterogeneous networks. In Proceedings of the IEEE $21^{s t}$ International. Conference on Telecommunications (ICT). Lisbon (Portugal), 2014, p. 236-240. DOI: 10.1109/ICT.2014.6845115

[29] ALAMOUTI, S. M. A simple transmit diversity technique for wireless communications. IEEE Journal on Selected Areas in Communications, 1989, vol. 16, no. 8, p. 1451-1458. DOI: $10.1109 / 49.730453$

[30] BASTOS, J., GAMEIRO, A. Performance of extended space-time coding techniques for MIMO MC-CDMA systems. In Proceedings of the IEEE First European Conference on Antennas and Propagation. Nice (France), 2006, 5 p. DOI: 10.1109/EUCAP.2006.4584673

\section{About the Authors ...}

Syed Saqlain ALI received his Masters degree in Telecommunication Engineering from the University of Trento, Italy in 2012. In December 2012 he started working towards the PhD Degree at Instituto de Telecomunicacões, Pólo de Aveiro, Portugal. His current research activities involve heterogeneous wireless networks, small-cell net- works, cognitive radio and interference mitigation techniques.

Daniel CASTANHEIRA received the Ph.D. degree in Electrical Engineering from the University of Aveiro, Aveiro, Portugal, in 2012. He is currently a Postdoctoral Researcher with the Instituto de Telecomunicações, Aveiro. He has been involved in several national and European projects, namely, PHOTON, within the FCT Portuguese National Scientific Foundation, and CODIV, FUTON, and QOSMOS with the FP7 ICT. In 2011, he was an Assistant Professor with the Departamento de Eletrónica, Telecomunicações e Informática, Aveiro University. His research interests lie in signal processing techniques for digital communications, with emphasis for physicallayer issues including channel coding, precoding/equalization, and interference cancelation.

Adão SILVA received the M.Sc. and Ph.D. degrees in Electronics and Telecommunications from the University of Aveiro, Aveiro, Portugal, in 2002 and 2007, respectively. He is currently an Assistant Professor with the Dept. of Electronics, Telecommunications and Informatics, University of Aveiro, and a Senior Researcher with the Instituto de Telecomunicações. He has been participating in several European research projects in the field of wireless networks. He has led several research projects, in the broadband wireless communications area, at the national level. His interests include multiuser MIMO, multicarrierbased systems, cooperative networks, precoding, and multiuser detection. Dr. Silva has published over 80 technical papers in international journals and conferences and acted as a member of the TPC of several international conferences.

Atilio GAMEIRO received the Licenciatura and the Ph.D. degrees from the University of Aveiro, Aveiro, Portugal, in 1985 and 1993, respectively. He is currently an Associate Professor with the Dept. of Electronics and Telecommunications, University of Aveiro, and a Senior Researcher with the Instituto de Telecomunicações, Pólo de Aveiro, where he is head of group. His industrial experience includes a period of one year at BT Labs and one year at NKT Elektronik. His main interests lie in signal processing techniques for digital communications and communication protocols, and within this research line, he has done work for optical and mobile communications, either at the theoretical and experimental level, and has published over 200 technical papers in international journals and conferences. His current research activities involve space-timefrequency algorithms for the broadband wireless systems and cross-layer design. He has been involved and has led IT or University of Aveiro participation on more than 20 national and European projects. 